\title{
Pflegepolitik jenseits des Koalitionsvertrags: Es gibt noch viel zu tun!
}

\section{HEINZ ROTHGANG, KLAUS JACOBS}

Prof. Dr. Heinz Rothgang ist Leiter der Abteilung Gesundheitsökonomie, Gesundheitspolitik und Versorgungsforschung des Zentrums für Sozialpolitik der Universität Bremen

Prof. Dr. Klaus Jacobs ist Geschäftsführer des Wissenschaftlichen Instituts der AOK (WIdO) in Berlin und Honorarprofessor an der Universität Duisburg-Essen

\author{
Langzeitpflege hat im Bundestagswahlkampf \\ eine erstaunlich große Rolle gespielt - selbst im \\ „Fernsehduell“ von Kanzlerin und Kanzlerkandidat war \\ sie eines der Hauptthemen. Verglichen damit sind die \\ Ergebnisse der Koalitionsverhandlungen eher mager \\ und lassen noch viel Raum für weitere notwendige \\ Maßnahmen, die im Koalitionsvertrag entweder gar \\ nicht thematisiert oder nur angedeutet wurden. Was \\ das heißt, soll im Folgenden verdeutlicht werden; \\ dabei wird der Schwerpunkt auf die Stichpunkte \\ Finanzierung und Leistungsdynamisierung gelegt.
}

\section{Finanzierung}

Die Zahl der Pflegebedürftigen wird in den nächsten Dekaden steigen, und das gilt entsprechend auch für die Ausgaben für Pflegebedürftigkeit. An dieser einfachen Feststellung führt kein Weg vorbei, die Frage ist nur, wie diese steigenden Ausgaben finanziert werden sollen. Im Koalitionsvertrag zwischen Union und FDP wurde hierzu 2009 behauptet: „In der Form der Umlagefinanzierung kann die Pflegeversicherung jedoch ihre Aufgabe, allen Bürgern eine verlässliche Teilabsicherung der Pflegekosten zu garantieren, auf Dauer nicht erfüllen. Daher brauchen wir neben dem bestehenden Umlageverfahren eine Ergänzung durch Kapitaldeckung, die verpflichtend, individualisiert und generationengerecht ausgestaltet sein muss" (CDU/CSU/ FDP 2009: 93). Im Ergebnis wurde bekanntlich die auch als "Pflege-Babr" bezeichnete staatlich geförderte ergänzende Pflegeversicherung eingeführt, allerdings nicht „verpflichtend“, sondern als freiwillige Option. Schon damit war sichergestellt, dass der Pflege-Bahr nur eine geringe Reichweite haben würde und nur einen kleinen Teil der Bevölkerung erreicht. Angesichts der massiven Kritik aus Politik, Wissenschaft (vgl. z. B. Jacobs/Rothgang 2012, 2013, Rothgang 2012a und b, Rothgang/Jacobs 2013) und von Seiten der Verbraucherschützer (Stiftung Warentest 2013; vzbv 2012) war fraglich, ob der Pflege-Bahr die Koalitionsverhandlungen überstehen würde. Dies galt umso mehr, als sein Namensgeber samt seiner Partei nicht mehr dem neuen Bundestag angehört.

Im Koalitionsvertrag gibt es zum Pflege-Bahr jedoch keine Aussage, sodass vom Fortbestand dieses fragwürdigen Instruments ausgegangen werden muss. Hilfreich dafür dürfte auch eine Kampagne gewesen sein, die die private Versicherungswirtschaft während der heißen Phase der Koalitionsverhandlungen lanciert hatte. Obwohl die Zahl der bisher erfolgten Vertragsabschlüsse mit 330.000 Ende Oktober 2013 bislang weit hinter den Annahmen der Bundesregierung zurückliegt - bei der Verabschiedung des Pflege-Neuausrichtungs-Gesetzes (PNG) Mitte 2012 war für 2013 von 
1,5 Mio. Abschlüssen ausgegangen und entsprechend ein Fördervolumen von 100 Mio. Euro in die Haushaltsplanung eingestellt worden -, gingen nicht nur viele Medien der Botschaft vom Pflege-Bahr als einem vermeintliche Verkaufsschlager auf den Leim; auch Jens Spahn, Chefverhandler der Union für Gesundheit und Pflege, erklärte, die große Nachfrage zeige, dass die Einführung richtig gewesen sei (Süddeutsche Zeitung vom 16.11.2013). Somit dürfte der Pflege-Bahr auf Dauer Teil der Pflegevorsorge bleiben. Angesichts seiner konzeptionellen Schwächen, insbesondere der Gefahr der Antiselektion, wird er aber kaum über ein Nischendasein hinausgelangen - insbesondere wenn auf eine Erhöhung der staatlichen

\section{Das größte Manko ist, dass keine Schritte zur Herstellung eines übergreifenden Solidarausgleichs zwischen privater und sozialer Pflegeversicherung vorgesehen sind.} Fördersätze verzichtet wird.

Ein Ausbau des Pflege-Bahr ist im Koalitionsvertrag allerdings auch nicht vorgesehen. Stattdessen soll die Kapitaldeckung zwar gesteigert werden, aber im Rahmen eines „Pflegevorsorgefonds“, der von der Bundesbank verwaltet wird (CDU/CSU/SPD 2013: 86). Gespeist werden soll dieser Fonds aus einer Beitragssatzsteigerung von 0,1 Beitragssatzpunkten. Nähere Ausführungen zu Zweck und Umsetzung des Fonds sind im Koalitionsvertrag nicht enthalten, sodass zur Interpretation auf die CDU-Position im Abschlussdokument der Arbeitsgruppe Gesundheit und Pflege zurückgegriffen werden muss. Dort heißt es: „Ziel muss es sein, insbesondere für den Zeitraum, in dem die geburtenstarke BabyboomerGeneration der 50er und 60er-Jahre in das typische Pflegealter kommt, vorzusorgen. Dies sind die Jahre 2035 bis 2055. Für diese Zeit wollen wir jetzt beginnen, eine Rücklage zu schaffen."

Diese Aussage ist gleich mehrfach problematisch. Tatsächlich steigt die Zahl der Pflegebedürftigen nach den gängigen Vorausberechnungen bis Mitte der 2050er Jahre an (Rothgang et al. 2011). Danach geht sie zwar wieder zurück, doch sinkt ebenfalls die Zahl der Beitragszahler. Es gibt also in Bezug auf den Beitragssatz keinen „Berg“, der „untertunnelt“ werden kann, sondern nur den Aufstieg auf ein „Hochplateau“ (Rothgang/Jacobs 2011). Was soll der Pflegevorsorgefonds also überhaupt bewirken und wie soll er funktionieren? Wenn das in den nächsten 20 Jahren angesammelte Kapital im Zeit- raum 2035 bis 2055 ausgegeben wird, ist der Fonds genau dann wieder leer, wenn die höchste Zahl an Pflegebedürftigen erreicht wird. Wird der Fonds dagegen ab 2035 nicht geleert, sondern sein Kapitalbestand (real) konstant gehalten, und nur die (Real-)Zinserträge werden in die Versorgung eingespeist, ergibt sich ab 2035 wieder ein reines Umlageverfahren. In der Zwischenzeit wurde dann staatlich zwangsgespart. Ob die gesamt-

vat

\section{(1)}

wirtschaftliche Sparquote dadurch beeinflusst wird oder lediglich private durch staatliche Ersparnis verdrängt wird, ist allerdings unklar. Sicher ist nur, dass es in Bezug auf intergenerative Gerechtigkeit sinnvoller wäre, die Staatsverschuldung (mit vergleichsweise hohen Sollzinsen) abzubauen, anstatt eine Nebenkasse (mit relativ niedrigen Habenzinsen) aufzubauen (Jacobs/Rothgang 2011).

Vielleicht besinnen sich der neue Gesundheitsminister Hermann Gröhe und sein Pflege-Bevollmächtigter Josef Laumann ja noch eines Besseren. Andernfalls werden kapitalfundierte Elemente bei der Pflegefinanzierung künftig gleich in zweifacher Hinsicht eine Rolle spielen: durch die Beibehaltung des Pflege-Bahr und die Einführung des Pflegevorsorgefonds. Schon in ihrem Koalitionsvertrag von 2005 hatten Union und SPD postuliert, dass „die Ergänzung des Umlageverfahrens durch kapitalgedeckte Elemente als Demografiereserve notwendig" sei (CDU/CSU/SPD 2005: 91). Umso bemerkenswerter ist es, dass eine andere Ankündigung von damals völlig in Vergessenheit geraten ist, denn 2005 gab es auch noch die Ankündigung: „Zum Ausgleich der unterschiedlichen Risikostrukturen wird ein Finanzausgleich zwischen gesetzlicher und privater Pflegeversicherung eingeführt“ (ebenda). Eine ähnliche Aussage gibt es heute nicht mehr, obwohl die unterschiedlichen Risikostrukturen unverändert fortbestehen. Die Versicherten der Privaten Pflegepflichtversicherung
(PPV) weisen gegenüber der Sozialen Pflegeversicherung (SPV) sowohl eine wesentlich günstigere Altersstruktur als auch deutlich niedrigere altersspezifische Pflegeprävalenzen auf (Rothgang et al. 2013: 53 ff.). Pro Versichertem werden daher - bei gleichem Leistungsanspruch und gleichen Bewilligungskriterien - in der PPV 75 Euro pro Jahr, in der SPV aber 300 Euro pro Jahr ausgegeben, also das Vierfache. Selbst wenn die zusätzlich anfallenden Beihilfeausgaben für knapp die Hälfte der Privatversicherten berücksichtigt werden, bleibt es dabei, dass pro SPV-Versichertem die zwei- bis dreifachen Leistungsausgaben anfallen. Hinzu kommt ein rund 60 \% höheres beitragspflichtiges Einkommen der Pri- Dass der aktuelle Koaten Unterschiede derart weit hinter den Vertrag von 2005 zurückfällt und keine Schritte zur Herstellung eines übergreifenden Solidarausgleichs vorsieht, muss als sein größtes Manko im Hinblick auf die Pflegefinanzierung angesehen werden. Gerade im Bereich der Pflegeversicherung, die 1994 nach Worten des Bundesverfassungsgerichts als „Pflegevolksversicherung in der Gestalt zweier Versicherungszweige“ (BVerfGE 103, 197) eingeführt wurde, wäre ein erster Schritt zum Ausgleich der Risikostrukturen und damit in Richtung auf ein integriertes Versicherungssystem möglich gewesen.

\section{Leistungsdynamisierung}

Anders als die Krankenversicherung ist die Pflegeversicherung bewusst als Teilleistungssystem konzipiert. Solange ihre nominal fixierten Leistungen nicht angehoben werden, verlieren sie angesichts steigender Pflegekosten real an Wert. Von 1993 bis 2007 wurden die Leistungen überhaupt nicht angepasst - mit der Folge eines Realwertverlusts von rund $20 \%$, der insbesondere anhand der steigenden Eigenfinanzierungsanteile im stationären Bereich gut nachvollziehbar ist (Rothgang et al. 2013: 121 ff.). Im Pflege-Weiterentwicklungsgesetz (PfWG) wurde erstmals eine Dynamisierung festgelegt, die inzwischen in drei Schritten (2008, 2010 und 2012) erfolgt ist. Allerdings war der Umfang dieser Anpassungen mit umgerechnet 1,4\% pro Jahr eher begrenzt (Rothgang et al. 2012: $31 \mathrm{ff}$.). Weiterhin wurde im neu gefassten $\mathbb{\$} 30$ 
SGB XI festgelegt: „Die Bundesregierung prüft alle drei Jahre, erstmals im Jahre 2014, Notwendigkeit und Höhe einer Anpassung der Leistungen der Pflegeversicherung. Als ein Orientierungswert für die Anpassungsnotwendigkeit dient die kumulierte Preisentwicklung in den letzten drei abgeschlossenen Kalenderjahren; dabei ist sicherzustellen, dass der Anstieg der Leistungsbeträge nicht höher ausfällt als die Bruttolohnentwicklung im gleichen Zeitraum. Bei der Prüfung können die gesamtwirtschaftlichen Rahmenbedingungen mit berücksichtigt werden“. Diese Regelung weist mehrere Schwächen auf: ihre Unverbindlichkeit („prüft Notwendigkeit“), die doppelte Begrenzung in Bezug auf Inflation und Bruttolohnentwicklung, die dafür sorgt, dass Reallohnsteigerungen, die sich in den Pflegekosten niederschlagen, regelmäßig zu Wertverlusten führen, und der Verweis auf die ,gesamtwirtschaftlichen Rahmenbedingungen“, der als Generalklausel für die Aussetzung oder Verringerung der Leistungsanpassungen genutzt werden.

Im Abschlussdokument der Arbeitsgruppe Gesundheit und Pflege war dennoch auf diese Regel Bezug genommen worden: „Pflege muss für alle Menschen, die auf sie angewiesen sind, bezahlbar bleiben. Wir wollen an der gesetzlich vorgesehenen Dynamisierung festhalten.“ Interessanterweise fehlt der zweite Satz jedoch in der endgültigen Version des Koalitionsvertrags. Dort heißt es nun an anderer Stelle: „Aus dieser Erhöhung stehen die Einnahmen von 0,2 Prozentpunkten zur Finanzierung der vereinbarten kurzfristigen Leistungsverbesserungen, insbesondere für eine bessere Betreuung der Pflegebedürftigen, sowie der für 2015 gesetzlich vorgesehenen Dynamisierung der Leistungen zur Verfügung“ (CDU/ CSU/SPD 2013: 86). Das Bekenntnis zu einer dauerhaften Dynamisierung, so schwach es schon war, fehlt im Koalitionsvertrag somit jetzt vollkommen. Für die notwendige Dynamisierung der Leistungen, die selbst keine Leistungsverbesserung darstellt, sondern ansonsten eintretende Leistungsverschlechterungen verhindert, steht danach noch ein „Restbetrag“ zur Verfügung, dessen Umfang von den vorgesehenen „echten“ Leistungsverbesserungen abhängt (etwa den an anderer Stelle explizit genannten Betreuungsleistungen bei stationärer Pflege). Damit dürfte der zur Verfügung stehende Betrag aber wahrscheinlich zu gering ausfallen, um einen weiteren Realwertverlust der Leistungen der Pflegeversicherung zu verhindern. Darüber hinaus fehlt jegliche langfristige Konzeption zur Leistungsdynamisierung. Soll die reale Kaufkraft der Pflegeversicherungsleistungen erhalten werden, ist eine entsprechende gesetzliche Vorschrift zu einer regelgebundenen Anpassung unabhängig von der Kassenlage erforderlich. Die Rürup-Kommission hatte zur Sicherstellung von Realwertkonstanz eine regelmäßige Anpassung nach einer Rate entsprechend dem Mittelwert aus Inflation und Bruttolohn- und -gehaltssteigerung gefordert. Weil zwei Drittel der Kosten von Pflegeeinrichtungen Personalkosten sind, wäre eine Formel, die die Anpassung als 2/3 der durchschnittlichen Bruttolohn- und -gehaltssteigerungen und 1/3 der Inflation berechnet, noch geeigneter. Eine solche regelgebundene Dynamisierung einzuführen, muss ein vorrangiges Ziel in diesem sozialen Sicherungssystem sein und hiervon ist der Koalitionsvertrag weit entfernt.

\section{Weitere Reformfelder}

Abschließend sollen noch kurz drei weitere Reformfelder angesprochen werden, bei denen die Ausführungen im Koalitionsvertrag ebenfalls unzureichend erscheinen: die Einführung eines neues Pflegebedürftigkeitsbegriffs, die Verhinderung eines drohenden Pflegenotstands sowie die Frage der künftigen Rolle der Kommunen.

Dass die bislang streng somatische Definition von Pflegebedürftigkeit die besonderen Belange der kognitiv beeinträchtigten Menschen nicht angemessen berücksichtigt, war schon bei Einführung der Pflegeversicherung erkennbar. Als Folge davon wird die Benachteiligung von Personen mit kognitiven Beeinträchtigungen und Verhaltensauffälligkeiten, insbesondere von Menschen mit Demenz, seit Ende der 1990er Jahre beklagt (vgl. Klie/Schmidt 1999; Sonntag/Angermeyer 2000; Wingenfeld 2000; Döhner/ Rothgang 2006). Dem ist die Politik zum einen begegnet, indem Personen mit eingeschränkter Alltagskompetenz in mehreren Gesetzen (PflegeleistungsErgänzungsgesetz 2002; PfWG 2008; PNG 2013) weitere Leistungen gewährt wurden, und zum anderen durch Einsetzung eines Beirats zur Überprüfung des Pflegebedürftigkeitsbegriffs bzw. zur konkreten Umsetzung des neuen Begriffs. Obwohl der Beirat 2009 zwei Berichte vorgelegt hat, alle Parteien diese Berichte begrüßt haben und deren Umsetzung Bestandteil der Ankündigungen des Koalitionsvertrags 2009 war, ist sie in der vergangenen Legislaturperiode nicht gelungen - sicherlich kein Ruhmesblatt der damaligen Koalition. Umso mehr ist zu hoffen, dass der neuerlichen Ankündigung nunmehr Taten folgen. Allerdings muss gleichzeitig vor übertriebenen Hoffnungen gewarnt werden (vgl. Rothgang 2013). Zum einen sind wichtige Fragen der Umsetzung erst noch zu klären, insbesondere die Leistungshinterlegung der neuen „Pflegebedarfsgrade“. Da dies nicht ganz einfach ist, erscheint der verhaltene Fahrplan des Koalitionsvertrags durchaus angebracht - wenn er denn umgesetzt wird. Zum anderen ist der neue Pflegebedürftigkeitsbegriff zunächst nur ein Instrument zur Zuteilung von Leistungsansprüchen - nicht mehr und nicht weniger. Er wird daher nicht alle Probleme der Pflegepolitik lösen, auch wenn dieser Anschein manchmal erweckt wird. Schließlich ist im Koalitionsvertrag angekündigt, bereits vor Umsetzung des neuen Pflegebedürftigkeitsbegriffs , die schon bestehenden Betreuungsleistungen weiter aus[zu]bauen und auf alle Pflegebedürftigen aus[zu] dehnen“. Damit werden dann erneut wie schon beim PNG - Leistungsrechte geschaffen, hinter die dann nur schwer wieder zurückgetreten werden kann und

\section{Soll die Kaufkraft der Pflegeversicherungsleistungen erhalten bleiben, ist eine regelgebunden Anpassung unabhängig von der Kassenlage notwendig.}

die zumindest für diejenigen, die diese Leistungen beziehen, bei Einführung des Pflegebedürftigkeitsbegriffs unter den Bestandsschutz fallen dürften, auch wenn de jure nur befristete Ansprüche eingeführt werden. Im Ergebnis führt dies entweder dazu, dass die Einführung des neuen Pflegebedürftigkeitsbegriffs sehr teuer wird oder dass sich Bestands- 
schutzregelungen nur auf die aktuellen Leistungsbezieher erstrecken und etwa zukünftige rein somatische Pflegebedürftige weniger Leistungen erhalten als ohne Reform. Es ist daher sehr die Frage, ob der Entschluss, zunächst zügig Leistungen auszubauen und dann erst den Pflegebedürftigkeitsbegriff umzusetzen, wirklich sinnvoll ist.

Entscheidend für die langfristige Weiterentwicklung der Langzeitpflege wird sein, inwieweit es gelingt, den sich abzeichnenden Engpass bei Beschäftigten in der Pflege zu schließen. Wie Berechnungen von Rothgang et al. (2012) gezeigt haben, ergibt sich unter der Annahme, dass sich die derzeitigen Trends fortsetzen, von 2009 bis 2030 eine (zusätzliche) Personallücke im Umfang von etwa einer halben Million Vollzeitbeschäftigen in der Pflege. Dem kann durch Verringerung des Bedarfs an Pflegekräften entgegengesteuert werden oder durch Ausbau der Beschäftigung. Ersteres würde auf stärkere Unterstützung pflegender Angehöriger, insbesondere durch Ausbau der Beratungskapazitäten und Maßnahmen zur besseren Vereinbarkeit von Pflege und Beruf, sowie die Mobilisierung zivilgesellschaftlicher Ressourcen hinauslaufen, Letzteres insbesondere auf die Erhöhung der Attraktivität des Pflegeberufs. Entsprechende Ansätze sind im Koalitionsvertrag erkennbar: So ist die geplante Zusammenlegung der Möglichkeiten von Pflegezeit- und Familienpflegezeitgesetz unter einem Dach mit Rechtsanspruch ebenso zu begrüßen wie der Prüfauftrag, die Anrechnungszeiten in der Rentenversicherung für pflegende Angehörige zu verbessern, und das Ziel, die Pflegeausbildung für alle Auszubildenden kostenfrei zu stellen. Andere Ankündigungen, wie die angestrebte „Aufwertung der Pflegeberufe“, bleiben jedoch vage. Hier wird sich erst bei der Umsetzung zeigen, welchen Wert diese Ankündigungen haben.

Die im Koalitionsvertrag vorgesehene Stärkung der Rolle der Kommunen, insbesondere im Hinblick auf ihre Planungs- und Steuerungskompetenz für die regionale Infrastruktur, ist grundsätzlich zu begrüßen, denn Pflege - auch das wird explizit betont - ist eine gesamtgesellschaftliche Aufgabe (CDU/
CSU/SPD 2013: 85). Gerade angesichts regional und lokal sehr unterschiedlicher zukünftiger Entwicklungen der Zahl der Pflegebedürftigen und der Pflegelandschaft (Rothgang et al. 2012), wurde eine stärkere Rolle der Kommunen auch im Vorwahlkampf vielfach gefordert (Engelmann et al. 2013; Hoberg et al. 2013). Dazu gehört aber auch eine entsprechende Finanzierungsbeteiligung der öffentlichen Haushalte. Davon ist im Koalitionsvertrag jedoch keine Rede etwa im Hinblick auf die angesprochene Entwicklung von Sozialräumen im $\mathrm{Zu}$ sammenwirken mit städtebaulichen Instrumenten. Allein schon die notwendigen qualifizierten personellen Ressourcen werden sich viele Kommunen angesichts ihrer prekären Finanzausstattung ohne substanzielle finanzielle Unterstützung gar nicht leisten können. Es bleibt zu hoffen, dass die geplante Bund-LänderArbeitsgruppe hierzu konkrete Vorschläge unterbreitet, die mehr beinhalten als den Vorschlag, die Sozialversicherung einmal mehr zur Finanzierung gesamtgesellschaftlicher Aufgaben zur Kasse zu bitten.

\section{Literatur}

CDU/CSU/FDP (2009): Wachstum. Bildung.

Zusammenhalt. Koalitionsvertrag zwischen CDU, CSU und FDP für die 17. Legislaturperiode vom 26.10.2009; Download: http:// www.cdu.de/sites/default/files/media/ dokumente/og1026-koalitionsvertrag-cducsufdp.pdf (Zugriff: 16.12.2013).

\section{CDU/CSU/SPD (2005): Gemeinsam für}

Deutschland. Mit Mut und Menschlichkeit. Koalitionsvertrag von CDU, CSU und SPD vom 11.11.2005; Download: www.cducsu.de/upload/ koavertrag0509.pdf (Zugriff: 16.12.2013).

CDU/CSU/SPD (2013): Deutschlands Zukunft gestalten. Koalitionsvertrag zwischen CDU,

CSU und SPD. 18. Legislaturperiode; Download: https://www.cdu.de/sites/default/files/ media/dokumente/koalitionsvertrag.pdf (Zugriff: 16.12.2013).

Döhner, H., Rothgang, H. (2006): Pflegebedürftigkeit. Zur Bedeutung der familialen Pflege für die Sicherung der Langzeitpflege, in: Bundesgesundheitsblatt - Gesundheitsforschung - Gesundheitsschutz, 49 (6): 583-594

Engelmann, D., Gohde, J., Künzel, G., Schmidt, S. (Redationsgruppe) (2013): Gute Pflege vor Ort. Das Recht auf eigenständiges Leben im Alter. Positionspapier im Auftrag der Abteilung Wirtschafts- und Sozialpolitik der Friedrich-Ebert-Stiftung. Berlin: FriedrichEbert-Stiftung.

Hoberg, R., Klie, T., Künzel, G. (2013): Strukturreform Pflege und Teilhabe; Download: http:// agp-freiburg.de/downloads/pflege-teilhabe/ Reformpaket_Strukturreform_PFLEGE TEILHABE_Langfassung.pdf (Zugriff: 16.12.2013). Jacobs, K., Rothgang, H. (2011): Zukunft der Pflegeversicherung. Eckpunkte für eine tragfähige Reform. G+G Wissenschaft, 11 (3): 14-21.

Jacobs, K., Rothgang, H. (2012): Der PflegeBahr: zum Schaden der Sozialen Pflegeversicherung und ihrer Versicherten. Gesundheitsund Sozialpolitik, 66 (5): 41-51.

\section{Jacobs, Klaus / Rothgang, Heinz (2013): Der} Pflege-Bahr: falsches Signal und untaugliches Geschäftsmodell. Replik zu Biederbick und Weber. Gesundheits- und Sozialpolitik, 67 (4): 24-27.

Klie, T.; Schmidt, R. (1999): Die Pflegeversicherung ist unter strategisch-politischen Gesichtspunkten ein großer Erfolg - allerdings besteht Reformbedarf. In: Theorie und Praxis der sozialen Arbeit, 50 (2): 48-53.

Rothgang, H. (2012a): Der "Pflege-Bahr" Umverteilung von unten nach oben. Soziale Sicherheit, 61 (6): 204

Rothgang, H. (2012b): Nach der Mini-Reform: Wesentliche Probleme bleiben ungelöst. Soziale Sicherheit, 61 (7): 245-254.

Rothgang, H. (2013): Der neue Pflegebedürftigkeitsbegriff - und seine Umsetzung. Soziale Sicherheit, 62 (7): 245-250.

Rothgang, H., Arnold, R., Wendlandt, K., Sauer, S., Wolter, A. (2011): Berechnungen der finanziellen Wirkungen verschiedener Varianten einer Pflegebürgerversicherung, Gutachten im
Auftrag der Bundestagsfraktion Bündnis $90 /$ Die Grünen, Zentrum für Sozialpolitik, Bremen; Download: http://www.zes.uni-bremen. de/lib/download. php?file=ade44659e4. pdf\&filename=ZeS-GA_2011_Gutachten_zur_ Pflegebuergerversicherung.pdf.

Rothgang, H., Jacobs, K. (2011): Substanziell und solidarisch - zur Zukunft der Pflegeversicherung. Gesundheits- und Sozialpolitik, 65 (4): 9-19.

Rothgang, H., Jacobs, K. (2013): Pflegereform 2014: Was ist zu tun? G+G Wissenschaft, 13 (3) 7-14

Rothgang, H., Müller, R., Unger, R. (2012): Themenreport „Pflege 2030“. Was ist zu erwarten was ist zu tun? Gütersloh: Bertelsmann-Stiftung.

Rothgang, H., Müller, R., Unger, R. (2013): BARMER GEK-Pflegereport 2013. Schwerpunktthema: Reha bei Pflege. Siegburg: Asgard-Verlag

Sonntag, A., Angermeyer, M. C. (2000): Zur Benachteiligung Demenzkranker im Rahmen der Pflegeversicherung. Zeitschrift für Sozialreform, 46 (12): 1048-1051

Stiftung Warentest (2013): Lieber ohne Förderung. Gute Pflegetagegeldversicherung können die Finanzlücke im Pflegefall schließen, die staatlich geförderte Vorsorge taugt aber wenig. Finanztest, Heft 5/2013: 70-77.

vzbv [Verbraucherzentrale Bundesverband] (2012): Die Zusatzversicherung für Pflege eine sinnvolle Ergänzung?; Download unter: www.vzbv.de/10919.htm (Zugriff: 16.12.2013). 